\title{
Pulmonary rehabilitation improves frailty and gait speed in some ambulatory patients with chronic lung diseases
}

\author{
Neha Mittal MD, Rishi Raj MD, Ebtesam Islam MD, PhD, Kenneth Nugent MD
}

\begin{abstract}
Objective: To determine the effect of rehabilitation on frailty markers in patients with chronic lung diseases.

Methods: Forty-one patients started pulmonary rehabilitation, and 30 patients completed at least 6 weeks of rehabilitation. Gait speed, weight loss, exhaustion, grip strength, and physical activity were assessed at the initial visit, at 6 weeks, and at 12 weeks to determine the frailty status using Fried's criteria.

Results: The study population (53\% women) had a mean age of $67 \pm 12$ years, a mean BMI of $27 \pm 9 \mathrm{~kg} / \mathrm{m} 2$, and a mean FEV1 of $50 \% \pm 17 \%$ of predicted. The average gait speed was $52.9 \pm 15.4 \mathrm{~m} / \mathrm{min}$; $17 \%$ were frail, $61 \%$ pre-frail, and $22 \%$ robust. Gait speed increased from $52.9 \pm 15.4$ meters per minute to $61.1 \pm 12.9$ meters per minute after 6 weeks $(P<0.01$ by paired $t$ test). The number of frail patients decreased from 5 to 2 after 6 weeks $(P=N S)$.

Conclusions: Gait speed increased in patients with chronic lung diseases with pulmonary rehabilitation. However, the frailty classification improved in some patients and declined in others.
\end{abstract}

Key words: Frailty, gait speed, pulmonary rehabilitation, COPD, outcomes

\section{INTRODUCTION}

Frailty is a distinct and complex syndrome that appears to represent a transitional state in the dynamic progression from robustness to functional decline. It results from multiple system impairments

Corresponding author: Neha Mittal, MD Contact Information: Neha.mittal@ttuhsc.edu DOI: 10.12746/swrccc2015.0312.151 occurring in conjunction with normal aging and is associated with sarcopenia, functional decline, neuroendocrine dysregulation, and immune impairment. ${ }^{1}$ Frailty has been studied for more than two decades, but a commonly accepted definition of frailty remains elusive. It results in disability, institutionalizations, and death. ${ }^{2}$ Physical exercise training involving balance, strength training, movement speed, and coordination has improved frailty markers in multiple randomized and nonrandomized studies. ${ }^{3-5}$ 
Chronic lung diseases, such as chronic obstructive pulmonary disease (COPD), were associated with frailty in the Cardiovascular Health Study. ${ }^{6-8}$ Inflammation, lack of activity or decreased exercise performance, and reduction in maximum oxygen uptake likely contribute to its development. ${ }^{9}$ Pulmonary rehabilitation improves skeletal muscle activity and exercise performance in patients with COPD. ${ }^{10}$ It also improves dyspnea, fatigue, emotional function, and patients' control over their medical conditions. ${ }^{11}$ Pulmonary rehabilitation would, therefore, be expected to improve frailty, but there are no published data on the effect of this intervention on frailty. The aim of this study was to determine the effects of pulmonary rehabilitation on frailty markers in community-dwelling patients with chronic lung diseases.

\section{Methods}

\section{Study Design}

This study measured the effect of pulmonary rehabilitation on frailty status and gait speed. Any patient with a chronic pulmonary disease, including COPD, asthma, pulmonary hypertension, and interstitial lung disease, was approached during the initial rehabilitation evaluation and asked to participate. The diagnoses were based on the medical records maintained by the referring physicians and/or hospital records, when available. The study participants represent a convenience sample; patients under the age of 21 or with acute respiratory illness were excluded from the study. Forty-one patients started the pulmonary rehabilitation program, 30 patients completed at least six weeks of rehabilitation, and 18 patients completed at least 12 weeks of rehabilitation. The study was approved by the Institutional Review Board at Texas Tech University Health Science Center (IRB\# L08-051); all patients gave written informed consent.

\section{Demographics}

Study investigators collected demographic information, including age, sex, ethnicity, body mass index (BMI), smoking history, medical history, home medications, oxygen use, pulmonary function test data, and history of recent falls and hospitalizations.
Falls and past hospitalization data were based on individual recall.

\section{Pulmonary Rehabilitation program}

These patients were referred for pulmonary rehabilitation by their physicians if they fulfilled Medicare criteria for enrollment into pulmonary rehabilitation and/or had "medical necessity" for rehabilitation based on physician judgment. Pulmonary rehabilitation included aerobic exercises using treadmills, bicycles, arm ergometers, and recumbent cross trainers and strength training using light weights, starting at a tolerable level on each machine and increasing the time as tolerated during two to three sessions per week. The usual goal was a total machine time between 40 and 50 minutes plus light upper body exercise with weights. Patients were educated on breathing retraining, disease physiology, beating the triggers in patients with obstructive airway disease, oxygen use and safety, preventing infections, relaxation techniques, nutritional counseling, pulmonary medications, and smoking cessation when applicable. Each rehabilitation session took about 60-90 minutes. The rehabilitation program is managed by a respiratory therapist with more than 10 years of work in pulmonary rehabilitation. Exercise sessions were also supervised by workers trained in exercise science and sports medicine. Additional details about this rehabilitation center are reported in other publications. ${ }^{12,13}$

\section{Outcomes}

The primary outcomes were to determine the effect of six weeks of pulmonary rehabilitation on frailty status and gait speed. Frailty was assessed using Fried's criteria. ${ }^{6}$ These criteria include weight loss, exhaustion, physical activity level using the Minnesota Leisure Time Activity Questionnaire, 15-foot walk time, and grip strength. One co-author $(\mathrm{EI})$ and one clinical research nurse from the Clinical Research Institute at Texas Tech University Health Sciences Center performed all evaluations. Both the 15-foot walk time used in Fried's criteria and the 100-foot walk time were measured. Grip strengths were measured with a JAMAR hydraulic hand dynamometer (Sammons 
Preston Roylan, Bolingbrook, IL); the best of three efforts with the dominant hand was recorded. Patients who met two Fried's criteria were classified as prefrail; patients who met three criteria were classified as frail.

The one hundred feet walk test was performed according to previously standardized protocol. ${ }^{14}$ The patient walked 50 feet in the hall, turned, walked 50 feet, and sat down. Patients were instructed to walk at their usual pace; time was recorded with a stop watch. Pulse rate, respiratory rate, and oxygen saturation $\left(\mathrm{SaO}_{2}\right)$ were recorded before the walk, after the walk, and after 3 minutes of recovery with a pulse oximeter. This 100-foot walk test was used because it causes a reproducible increase in heart rate in healthy subjects and therefore creates a mild physiological stress. In addition, it has satisfactory repeatability and provides an opportunity to evaluate the physiological cost index. ${ }^{15}$

Patients who participated in rehabilitation performed additional 100-foot walk tests and underwent additional frailty assessments at 6 and 12 weeks. Patients who had a second follow-up at 5-7 weeks were counted in the 6 week follow-up group, and patients with a third follow-up at 10-13 weeks were counted in the 12 week follow-up group for gait speed measurements and frailty assessment. Some patients missed scheduled follow-up assessments due to intercurrent illness.

\section{Statistical Analysis}

Data were analyzed using Statistical Package for Social Sciences V.19.0.0 (SPSS, IBM Inc., USA). Chi square analysis of contingency table was used to assess group differences for discreet variables and Fisher's exact test was used when each cell had expected frequency of less than 5. Parametric tests ( $T$ test, ANOVA), and non-parametric tests (KruskalWallis test) were used to assess group differences for continuous variables. $P$ value of $<0.05$ was considered statistically significant.

\section{RESULTS}

\section{STUDY POPULATION CHARACTERISTICS}

Forty-one patients started pulmonary rehabilitation; 30 patients completed at least 6 weeks of rehabilitation, and 18 patients completed 12 weeks of rehabilitation. Baseline demographic characteristics of the 30 patients are detailed in Table 1. Fifty-three percent of subjects were female. The mean age was $67 \pm 12$ years (range: $38-85$ years), and the mean body mass index was $27 \pm 9 \mathrm{~kg} / \mathrm{m} 2$ (range: $15-61 \mathrm{~kg} /$ $\mathrm{m} 2$ ). Pulmonary diagnoses included chronic obstructive pulmonary disease $(77 \%)$, asthma $(30 \%)$, pulmonary fibrosis $(10 \%)$, and pulmonary hypertension (7\%). The mean $\mathrm{FEV}_{1}$ was $50 \pm 17 \%$ of predicted (range: $26-85 \%$ predicted). Other co-morbidities included congestive heart failure (13\%), cerebrovascular disease (10\%), coronary artery disease (33\%), peripheral vascular disease (0\%), diabetes mellitus type $2(13 \%)$, hypertension $(40 \%)$, renal disease $(0 \%)$, and arthritis (20\%). On the initial evaluation, $17 \%$ subjects were frail, $61 \%$ were pre-frail, and $22 \%$ were robust. These patients had frequent falls and hospitalizations within the last one year (Table 1).

\section{Changes in frallty and gait SPEed after PULmonary Re- HABILITATION.}

Thirty patients completed 6 weeks of rehabilitation but only 29 had frailty evaluations at week 1 and at week 6 . Eighteen patients had a third frailty evaluation at week 12; some patients (12) did not have a final evaluation due to scheduling conflicts or intercurrent illness precluding attendance. There were fewer frail patients after 6 weeks of rehabilitation in the 29 patients who had evaluations at the start of rehabilitation and after 6 weeks of rehabilitation (7\% at 6 weeks vs. $17 \%$ at 1 week; Table 2). There were also fewer frail patients after 6 weeks of rehabilitation in the 18 patients who completed 12 weeks of rehabilitation ( $6 \%$ at 6 weeks vs. $17 \%$ at 1 week; Table 3 ). There were no statistically significant differences in the frailty status in the 1-6 weeks participation cohort and the 1-6-12 weeks participation cohort by Fischer exact tests. Five patients had a deterioration in their frailty status during rehabilitation; four patients from week 1-6 group (29 total) went from robust to prefrail, and one patient from week 6-12 group (18 total) went from pre-frail to frail. Review of the rehabilitation center files did not identify any definite explanations for these changes in status. 
Table 1 Baseline characteristics of the 30 patients completing six weeks of rehabilitation

\begin{tabular}{|c|c|}
\hline Characteristics & Mean \pm SD (Range), or Percent (\%) \\
\hline Age (years) & $67 \pm 12(38-85)$ \\
\hline BMI & $27 \pm 9(15-61)$ \\
\hline $\mathrm{FEV}_{1}(\%)$ & $50 \pm 17(26-85)$ \\
\hline Gender: Female & $53 \%$ \\
\hline Ethnicity: White & $86.7 \%$ \\
\hline Hispanic & $10.0 \%$ \\
\hline Black & $0.0 \%$ \\
\hline Asian & $0.0 \%$ \\
\hline Other & $3.3 \%$ \\
\hline Tobacco use: Current & $16.7 \%$ \\
\hline Past & $70.0 \%$ \\
\hline None & $13.3 \%$ \\
\hline \multicolumn{2}{|l|}{ Pulmonary diagnoses } \\
\hline COPD* & $77 \%$ \\
\hline Asthma* & $30 \%$ \\
\hline Pulmonary Fibrosis & $10 \%$ \\
\hline Pulmonary HTN & $7 \%$ \\
\hline \multicolumn{2}{|l|}{ Comorbidities } \\
\hline $\mathrm{CHF}$ & $13 \%$ \\
\hline Stroke & $10 \%$ \\
\hline CAD & $33 \%$ \\
\hline PVD & $0 \%$ \\
\hline Hypertension & $40 \%$ \\
\hline Diabetes mellitus & $13 \%$ \\
\hline Renal insufficiency & $0 \%$ \\
\hline Osteoarthritis & $20 \%$ \\
\hline Oxygen use: None & $37 \%$ \\
\hline At Night & $17 \%$ \\
\hline Continuous & $47 \%$ \\
\hline Falls in last one year: None & $70 \%$ \\
\hline 1 to 5 & $23 \%$ \\
\hline$>5$ & $7 \%$ \\
\hline Falls in the last 3 months: None & $83 \%$ \\
\hline 1 to 5 & $17 \%$ \\
\hline$>5$ & $0 \%$ \\
\hline Hospitalization in last 1 year: None & $33 \%$ \\
\hline Once & $43 \%$ \\
\hline$>1$ & $23 \%$ \\
\hline
\end{tabular}


Table 1 Baseline characteristics of the 30 patients completing six weeks of rehabilitation (Continued)

\begin{tabular}{|c|l|}
\hline Characteristics & Mean \pm SD (Range), or Percent (\%) \\
\hline Frailty status: Robust* & $17 \%$ \\
\hline Pre-frail & $61 \%$ \\
\hline Frail & $22 \%$ \\
\hline
\end{tabular}

"Some patients had the diagnosis of both COPD and asthma,

** Percent is based on 29 patients who completed 6 weeks of rehabilitation

CHF- congestive heart failure, PVD- peripheral vascular disease,

CAD-coronary artery disease

Table 2 Frailty data for 29 patients who finished 6 weeks of rehab

\begin{tabular}{|c|c|c|c|c|}
\hline \begin{tabular}{|l} 
Week 1 \\
Week $6^{*}$
\end{tabular} & Frail & Pre-frail & Robust & $\begin{array}{l}\text { Total } \\
\text { (Week 6) }\end{array}$ \\
\hline Frail & 2 & 0 & 0 & $2(7 \%)$ \\
\hline Pre-frail & 3 & 15 & 4 & $22(76 \%)$ \\
\hline Robust & 0 & 2 & 3 & $5(17 \%)$ \\
\hline $\begin{array}{l}\text { Total } \\
\text { (Week 1) }\end{array}$ & $5(17 \%)$ & $17(59 \%)$ & $7(24 \%)$ & $29(100 \%)$ \\
\hline
\end{tabular}

"There was no difference in the distribution between week 1 and week 6 based on Fischer exact tests using all three categories or using two categories with frail and pre-frail as a single group. 
Table 3 Frailty status of 18 patients at weeks 1, 6, and 12 of rehabilitation

\begin{tabular}{|c|c|c|c|c|}
\hline 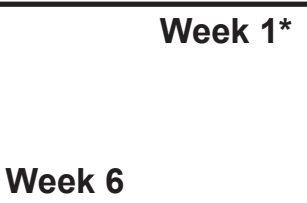 & Frail & Pre-frail & Robust & $\begin{array}{l}\text { Total } \\
\text { Week } 6\end{array}$ \\
\hline Frail & 1 & 0 & 0 & $1(6 \%)$ \\
\hline Pre-Frail & 2 & 10 & 2 & $14(78 \%)$ \\
\hline Robust & 0 & 1 & 2 & $3(17 \%)$ \\
\hline $\begin{array}{l}\text { Total } \\
\text { Week } 1\end{array}$ & $3(17 \%)$ & $11(61 \%)$ & $4(22 \%)$ & $18(100 \%)$ \\
\hline $\begin{array}{l}\text { Week 6** } \\
\text { Week } 12 \\
\end{array}$ & Frail & Pre-frail & Robust & $\begin{array}{l}\text { Total } \\
\text { Week } 12\end{array}$ \\
\hline Frail & 0 & 1 & 0 & $1(6 \%)$ \\
\hline Pre-Frail & 1 & 8 & 0 & $9(50 \%)$ \\
\hline Robust & 0 & 5 & 3 & $8(44 \%)$ \\
\hline $\begin{array}{l}\text { Total } \\
\text { Week } 6\end{array}$ & $1(6 \%)$ & $14(78 \%)$ & $3(17 \%)$ & $18(100 \%)$ \\
\hline
\end{tabular}

${ }^{*, * *}$ There are no differences in the distribution between week 1 and week 6 or between week 6 and week 12 based on Fischer exact tests using all three categories or using two categories with frail and pre-frail as a single group.

Thirty-seven patients had gait speed measurements at week 1 (initial evaluation) and at week 6 , and 22 of these patients had another gait speed measurement at week 12 (some of these patients in both groups missed scheduled frailty evaluations). For the 37 patients undergoing gait speed testing at week 1 and at week 6 of rehabilitation, gait speed increased from $52.9 \pm 15.4$ meters per minute at week 1 to $61.1 \pm$ 12.9 meters per minute at week $6(p=0.001$, paired t-test). The mean increase was $8.4 \pm 13.9$ meters per minute ( $95 \% \mathrm{Cl}$ - 3.8 meters per minute to 13.0 meters per minute, $p=0.0001$, paired t-test) (Figure 1). Twenty-two patients continued rehabilitation for an additional six weeks. They had no additional increase in gait speeds during the second six weeks of rehabilitation. Mean gait speed for this group was $63.2 \pm 11.4$ meters per minute at 6 weeks and $62.5 \pm 12.7$ meters per minute at 12 weeks ( $p=0.72$, paired t test) (Figure $1)$. 


\section{Gait speed change with rehabilitaton}

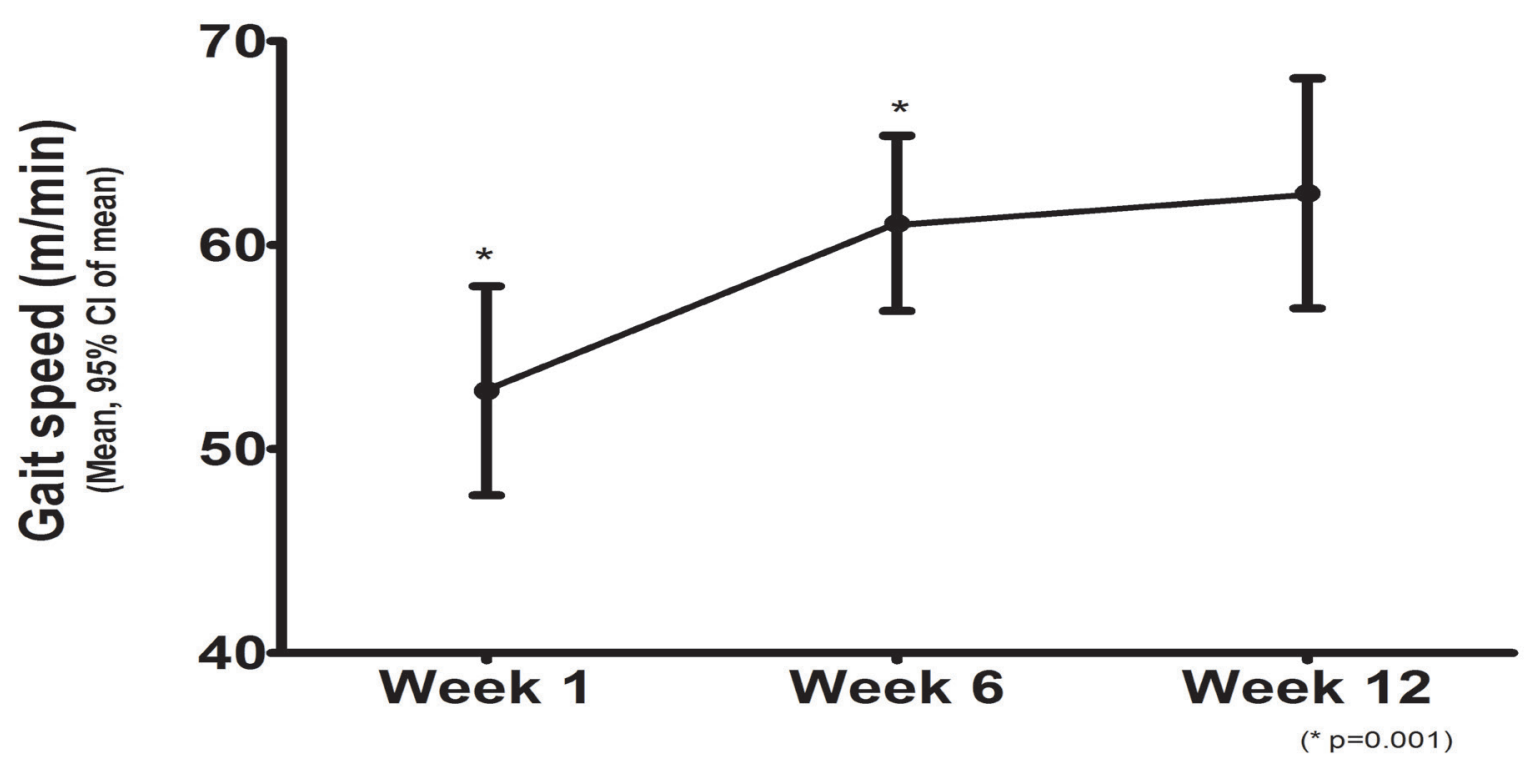

Figure $1 \mathrm{Gait}$ speed $(\mathrm{m} / \mathrm{min}$ ) before and after six and twelve weeks of rehabilitation for all patients (37 at 6 weeks and 22 at 12 weeks). Gait speed increases after rehabilitation ( $p=0.002$, paired sample t test) for all patients. Gait speed increases by 8.2 meters per minute overall $(95 \% \mathrm{Cl}$ for change $3.5-13.6$ meters per minute).

\section{Discussion}

This study demonstrates that pulmonary rehabilitation improves both gait speed and frailty status in some but not all ambulatory patients with chronic lung diseases. Most of the improvement in gait speed occurred in the first six weeks of rehabilitation. The proportion of frail patients decreased, and the proportion of robust patients increased with rehabilitation. However, pulmonary rehabilitation did not have a uniform effect in this small study cohort. Some patients had deterioration in their frailty status without a definite explanation.

Frailty has been associated with several chronic diseases and predicts worse outcomes. The Cardiovascular Health Study (CHS) showed an association between frailty and cardiovascular disease (odds ratio 7.5 in patients with $\mathrm{CV}$ disease compared to healthy controls).$^{16}$ Fried et al used data from the Cardiovascular Health Study and observed a close asso- ciation between frailty status and incident falls, worsened mobility, incident hospitalization, and death over 3 or 7 years. 6 Park determined the frequency of frailty in patients using data from the National Health and Nutrition Evaluation Survey dataset (2003-2006). ${ }^{17}$ Based on these survey responses, these investigators concluded that $57.8 \%$ of patients with COPD were frail. Galizia et al studied mortality in elderly subjects with COPD and frailty. Over a 12 year follow-up frail subjects with COPD had a higher mortality rate than frail subjects without COPD. ${ }^{18}$ Subjects with increasing frailty scores had increased mortality. The prevalence of frailty and pre-frailty in our study likely reflects the characteristics of patients seen in pulmonary rehabilitation centers. Our patients had frequent self-reported hospitalizations and falls and slower gait speeds as reported in other studies. ${ }^{6-8,19-22}$ Frailty has been associated with increased risk for falls in other disease processes. The association of frailty with poor health outcomes in patients with chronic diseases has important clinical implications. 
Rehabilitation with balance training and muscle strengthening has improved phenotypic markers of frailty in small studies which used varying definitions of frailty. ${ }^{3-5,6,23,24}$ The current literature on the effects of interventions on frailty includes studies on physical rehabilitation using resistance training, balance exercises, and aerobic training. ${ }^{25,26}$ All these studies showed positive effects of exercise on various characteristics of frailty, but there is no study that shows an overall change/reversal in frailty status with pulmonary rehabilitation based on Fried's criteria. ${ }^{3-5,3,24,27} \mathrm{~A}$ meta-analysis published by Lacasse and coworkers studied the impact of pulmonary rehabilitation on four major domains of quality of life (chronic respiratory questionnaire scores of dyspnea, fatigue, emotional function, and mastery) in COPD patients and found statistically significant improvement in all the outcomes. ${ }^{11}$ The ongoing Frailty Intervention Trial will try to determine the impact of multiple interventions on frailty in pre-frail and frail community-dwelling older adults. ${ }^{28}$ Our study demonstrates that pulmonary rehabilitation improves frailty in some patients, but this effect was not consistent.

\section{LIMITATIONS}

Our study is limited by a relatively small number of patients completing more than six weeks of rehabilitation and by the missed scheduled evaluations due intercurrent illness. In addition, we could not determine why some patients had a decline in frailty status; this could reflect intercurrent illness, the effect of other co-morbidity, or the progression of their lung disease even though they were in rehabilitation. We used only one frailty assessment tool; the physical activity component of this tool requires time to complete and provides only estimates about activity levels. Studies like this need a robust tool with less dependence on subjective responses. Finally, we did not compare changes in frailty status with other outcomes, such as changes in the quality of life.

\section{Conclusion}

Frailty is common in patients with chronic lung diseases, and slow gait speed is a good indicator for frailty in these patients. Both frailty and gait speed improve in some patients with pulmonary rehabilitation.

\section{ACKNoWLedgements}

We would like to thank Lori Stroud, CRT, RCP, at the Rehabilitation Center for providing constant support and help with patient enrollment. Chris ScottJohnson MSN, RN, C, CCRC at the Clinical Research Institute at Texas Tech University Health Sciences Center helped with patient enrollment and assessment.

Author Affiliation: Neha Mittal, Ebtesam Islam, and Kenneth Nugent work in the Department of Internal Medicine at Texas Tech University Health Sciences Center in Lubbock, TX. Rishi Raj works in the Department of Internal Medicine at Northwestern University in Chicago, ILL.

Received: 04/21/2015

Accepted: 09/21/2015

Reviewers: Dean Diersing MS, John Culberson MD

Published electronically: 10/15/2015

Conflict of Interest Disclosures: None

\section{REFERENCES}

1. Lang PO, Michel JP, Zekry D. Frailty syndrome: a transitional state in a dynamic process. Gerontology 2009; 55:539549

2. Speechley M, Tinetti M. Falls and injuries in frail and vigorous community elderly persons. J Am Geriatr Soc 1991; 39:46-52

3. Binder EF, Schechtman KB, Ehsani AA, et al. Effects of exercise training on frailty in community-dwelling older adults: results of a randomized, controlled trial. J Am Geriatr Soc 2002; 50:1921-1928

4. Gill TM, Baker DI, Gottschalk M, Peduzzi PN, Allore H, Byers A. A program to prevent functional decline in physically frail, elderly persons who live at home. N Engl J Med 2002; 347:1068-1074

5. Chin A Paw MJM, van Uffelen JGZ, Riphagen I, Van Mechelen W, . The Functional Effects of Physical Exercise TRaining in Frail Older People. A Systematic Review. . Sports Medicine 2008; 38:781-793

6. Fried LP, Tangen CM, Walston J, et al. Frailty in older adults: evidence for a phenotype. J Gerontol A Biol Sci Med Sci 2001; 56:M146-156 
7. Bandeen-Roche K, Xue Q, Ferrucci L, et al. Phenotype of frailty: Characterization in the Women's health and Aging Studies. Journal of Gerontology Series A: Biological Sciences and Medical Sciences 2006; 61:262-266

8. Thorpe RJ, Jr., Weiss C, Xue Q, Fried LP. Transitions among disability levels or death in African American and white older women. J Gerontol A Biol Sci Med Sci 2009; 64:670-674

9. Nussbaumer-Ochsner Y, Rabe KF. Systemic manifestations of COPD. Chest 2011; 139:165-173

10. Nici L, Donner C, Wouters E, et al. ATS/ERS Pulmonary Rehabilitation Writing Committee. American Thoracic Society/ European Respiratory Society Statement on Pulmonary Rehabilitation American Journal of Respiratory and Critical Care Medicine 2006; 173:1390-1413

11. Lacasse Y, Martin S, Lasserson TJ, Goldstein RS. Metaanalysis of respiratory rehabilitation in chronic obstructive pulmonary disease. A Cochrane systematic review. Eura Medicophys 2007; 43:475-485

12. McClellan R, Amiri HM, Limsuwat $C$, Nugent KM. Pulmonary rehabilitation increases gait speed in patients with chronic lung diseases. Health Services Research and Managerial Epidemiology 2014:1-5, doi: $10.177 / 2333392814533659$

13. Amiri HM, McClellan R, Limsuwat $C$, Nugent $K$. Exercise duration during pulmonary rehabilitation: an index of efficacy. The Southwest Respiratory and Critical Care Chronicles 2013; 1(4):3-7.

14. Raj R, Guerra D, Sehli S, et al. One hundred-foot walk test for functional assessment of clinic patients. Am J Med Sci 2009; 338:361-367

15. Raj R, Amiri HM, Wang H, Nugent KM. The repeatability of gait speed and physiological cost index measurements in working adults. Jour of Prim Care and Comm Health 2014;5(2):128-133.

16. Newman AB, Gottdiener JS, McBurnie MA, et al. Associations of subclinical cardiovascular disease with frailty. Journal of Gerontology Series A: Biological Sciences and Medical Sciences 2001; 56:M158-166

17. Park SK, Richardson CR, Holleman RG, Larson JL. Frailty in people with COPD, using the national health and nutrition evaluation survey dataset (2003-2006). Heart Lung 2013; 42(3):163-170.

18. Galizia G, Cacciatore F, Testa G, Della-Morte D, Mazzella F, Langellotto A, Raucci C, Gargiulo G, Ferrara N, Rengo F, Abete P. Role of clinical frailty on long-term mortality of elderly subjects with and without chronic obstructive pulmonary disease. Aging Clin Exp Res 2011 Apr; 23(2):118-125.

19. Walston J, McBurnie MA, Newman A, et al. Frailty and activation of the inflammation and coagulation systems with and without clinical comorbidities: results from the Cardio- vascular Health Study. Arch Intern Med 2002; 162:23332341

20. Rothman MD, Leo-Summers L, Gill TM. Prognostic significance of potential frailty criteria. J Am Geriatr Soc 2008; 56:2211-2116

21. Abellan van Kan G, Rolland Y, Andrieu S, et al. Gait speed at usual pace as a predictor of adverse outcomes in community-dwelling older people. An International Academy on Nutrition and Aging (IANA) Task Force. Journal of Nutrition, Health and Aging 2009; 13:881-889

22. Buchman AS, Wilson RS, Boyle PA, Bienias JL, Bennett DA. Change in motor function and risk of mortality in older persons. J Am Geriatr Soc 2007; 55:11-19

23. Peterson MJ, Giuliani C, Morey MC, et al. Physical activity as a preventative factor for frailty: the health, aging, and body composition study. J Gerontol A Biol Sci Med Sci 2009; 64:61-68

24. Hubbard RE, Fallah N, Searle SD, Mitnitski A, Rockwood K. Impact of exercise in community-dwelling older adults. PLoS One 2009; 4:e6174

25. Latham NK, Bennett DA, Stretton CM, Anderson CS. Systematic review of progressive resistance strength training in older adults. J Gerontol A Biol Sci Med Sci 2004; 59:48-61

26. Liu CJ, Latham NK. Progressive resistance strength training for improving physical function in older adults. Cochrane Database Syst Rev 2009:CD002759

27. Hardy SE, Perera S, Roumani YF, Chandler JM, Studenski SA. Improvement in usual gait speed predicts better survival in older adults. J Am Geriatr Soc 2007; 55:1727-1734

28. Fairhall N, Aggar C, Kurrle SE, et al. Frailty Intervention Trial (FIT). BMC Geriatr 2008; 8:27 